\title{
Metabolic Nutri-Expert System: A Comprehensive Tool for Achieving Metabolic Control of Inborn Errors of Amino Acid Metabolism
}

\author{
Zohreh Mazloom \\ Department of Clinical Nutrition, \\ School of Nutrition and Food \\ Sciences \\ Shiraz University of Medical \\ Sciences, Shiraz, Iran
}

\author{
Seyedeh Maryam \\ Abdollahzadeh \\ Department of Clinical Nutrition, \\ School of Nutrition and Food \\ Sciences \\ Shiraz University of Medical \\ Sciences, Shiraz, Iran
}

\author{
Mojtaba Zandieh \\ MSc in Artificial Intelligence, \\ Shiraz, Iran
}

\author{
Vahid Ashktorab \\ MSc in Computer Software \\ Engineering, Shiraz, Iran
}

\author{
Baran Hashemi \\ Rajaie Cardiovascular, Medical \\ and Research Center \\ Iran university of Medical \\ Sciences, Tehran, Iran
}

\author{
Zohreh Karamizadeh \\ Department of Pediatrics \\ Shiraz University of Medical Sciences, Shiraz, Iran
}

\author{
Hossein Moravej \\ Neonatal research center \\ Shiraz University of Medical \\ Sciences, Shiraz, Iran
}

\begin{abstract}
The main aim of this study was to develop a comprehensive nutritional planning expert system for inborn errors of amino acid metabolism (IEAAM). Metabolic Nutri-Expert System, integrated in the electronic nutritional history record, was designed to accelerate either dietitian or patient/parent knowledge acquisition and education about the particular disease and propose culturally appropriate low protein diet to improve metabolic control or maintain the present health status. The Genetic Metabolic Dietitians International (GMDI) nutritional guideline was used to estimate the recommended nutritional values in the proposed system. The recommended daily Intakes (RDIs) for patients ingesting amino acid mixture, the U.S. Department of agriculture (UDSA) nutrient list, and both Iranian and modern low protein recipes were applied to perform diet planning. The proposed system also allows the user to modify the diet as he/she likes, and to propose his/her own low protein cookery recipes as well. This comprehensive computer system, through the multidisciplinary viewpoint, aimed to at least partially replace some of the regular traditional metabolic dietitian visits and optimize the patient's metabolic control and adherence outcomes.
\end{abstract}

\section{General Terms \\ Expert System}

\section{Keywords}

Inborn errors of metabolism; Expert systems; Nutrition Therapy; Amino acids

\section{INTRODUCTION}

Inborn errors of metabolism (IEM) are a large group of rare genetic disorders which involve a disruption in metabolic pathways of macronutrients (carbohydrate, fat, and protein).
Among them are inborn errors of amino acid (blocks of protein) metabolism (IEAAM), which are characterized by high blood concentration of one or more amino acids, and are assumed to result from deficient activity of an enzyme responsible in protein metabolism [1]. IEAAM has been neglected over the years in Iran, like many other developing countries, since although collectively common, they are individually unusual $[1,2]$. Due to advances in medical technology, characterization of human genome, and expanded newborn screening (NBS) programs using tandem mass spectrometry (MS/MS), IEAAM are nowadays more often detected [3-5].

Along with both acute and chronic medical management, targeted metabolic nutrition therapy remains the cornerstone of treatment for IEAAM [6]. Provision of adequate energy and protein, restriction of disorder-specific amino acid(s) (DSAA), supplementation of either coenzyme or the conditionally essential amino acid(s) alone or in combination, and removal of toxic metabolites are the prominent principles of nutrition management for affected individuals with IEAAM [7]. Physician, metabolic dietitian and affected patient (or his/her parent/caregiver) should, therefore, adequately communicate to maintain adequate metabolic control of the disease $[3,8]$.

Although the efficacy of parental involvement in improving various aspects of chronic disease management was previously revealed $[9,10]$, dietary management of IEEMs was reported as the biggest challenge faced by parents to overcome [11]. On the other hand, promoting selfmanagement of such disorders through increasing the individual's knowledge and self-esteem was revealed to play a crucial role in obtaining adequate metabolic control $[12,13]$.

Nowadays, an increasing number of either computer or web- 
based software/systems are available in the medical field and clinical setting. The introduction of artificial intelligence into clinical nutrition leads to the ease of implementation of disorder-specific nutritional information in practice. Up to now, only a few software have been developed with the goal of enabling both patient (http://www.metabolicdietapp.org) and metabolic dietician (http://www.metabolicpro.org) to reach adequate metabolic control and to design an appropriate disorder-specific low-protein diet in several IEAAM

On the other hand, the dietary culture of each country is a collection of traditional dietary- and food consumption patterns dependent on the natural environment and available foodstuff [14]. Iranian culinary culture is very different from those of western countries; the recipes included in the mentioned software are, therefore, not much acceptable by Iranian patients. Thus, the inclusion of local dishes may be helpful to combat the problem.

Metabolic Nutri-Expert System is developed for prescription of a culturally suitable DSAA-restricted diet component by component through the predesigned database, creating alert in case of selection of lower or higher value of intended diet components, and recording the composition of diet for a diverse set of IEEM. The application provides disorderspecific nutrient recommendations based upon anthropometric and biochemical parameters, food intake analysis, design of "sick-day"- and "usual"-diet, and proper nutritional recommendation. In the present paper, Metabolic Nutri-Expert System is described for phenylketonuria (PKU), as an example of IEAAM, following the brief explanation of the expert system.

\section{EXPERT SYSTEM}

An expert system is a computer-based problem-solving system used to simulate solutions of genuine experts when facing a real challenge. Indeed, medical expert system empowers the user to make much more informed medical decision and, thus, improve health care quality. The major components of an expert system are: 1) the knowledge base, i.e. domain knowledge comprised of a set of facts and rules provided by human expert, 2) interference engine as the processor of the system which uses the knowledge base to draw a conclusion or (if not available) the facts obtained from the user to supplement the rules, and 3) user interface as the component to interact with the final user.

Metabolic Nutri-Expert System is developed by C Language Integrated Production System (CLIPS) language, a shell providing a comprehensive structure for a forward chaining rule-based expert system.

\section{METABOLIC NUTRI-EXPERT SYSTEM}

The proposed Metabolic-Nutri Expert System is developed to help the user improve his/her knowledge and nutritional skill levels in order to achieve and maintain adequate metabolic control and optimal intellectual outcome. It also facilitates dealing with several emergencies (ex: 'sick day' management, and decrease or increase the levels of disorder-specific blood parameters). The Metabolic-Nutri Expert System can be applied for dietary management of several IEAAM, the list of which and their associated amino acids are shown in Table 1. The system is comprised of numerous subsets (See Figure 1).

Table 1: IEAAM provided in the proposed expert system and their related DSAA

\begin{tabular}{|c|c|c|}
\hline IEAAM & Abbreviation & DSAA \\
\hline Phenylketonuria & PKU & Phenylalanine (PHE) \\
\hline Tyrosinemia & - & Tyrosine (TYR) \\
\hline Maple syrup urine disease & MSUD & $\begin{array}{l}\text { Leucine (LEU), Isoleucine (ILE), Valine } \\
\text { (VAL) }\end{array}$ \\
\hline Isoaleric academia & IVA & Leucine (LEU) \\
\hline$\beta$-methylcrotonyl-CoA carboxylase deficiency & $\beta \mathrm{MCC}$ deficiency & Leucine (LEU) \\
\hline $\begin{array}{l}\beta \quad \text {-hydroxy- } \beta \text {-methylglutaryl-CoA lyase } \\
\text { deficiency }\end{array}$ & HMG-CoA lyase deficiency & Leucine (LEU) \\
\hline $\begin{array}{l}\beta \text {-methylglutaconyl-CoA hydratase deficiency ( } \beta \\
- \text { Methylglutaconic aciduria ( } \beta \text { MGA)-Type I) } \dagger\end{array}$ & $\beta M G A$ Type I & Leucine (LEU) \\
\hline $\begin{array}{l}\text { Mitochondrial acetoacetyl-CoA thiolase }(\beta- \\
\text { ketothiolase deficiency) }\end{array}$ & BKT deficiency & Isoleucine (ILE) \\
\hline $\begin{array}{l}\alpha \text {-Methyl- } \beta \text {-hydroxybutyryl-CoA dehydrogenase } \\
\text { deficiency } \dagger\end{array}$ & MHBD deficiency & Isoleucine (ILE) \\
\hline Propionic academia & PPA or PA & $\begin{array}{l}\text { Isoleucine (ILE), Valine (VAL), } \\
\text { Methionine (MET), Threonine (THR) }\end{array}$ \\
\hline Methylmalonic academia & MMA & $\begin{array}{l}\text { Isoleucine (ILE), Valine (VAL), } \\
\text { Methionine (MET), Threonine (THR) }\end{array}$ \\
\hline Glutaric academia type-I & GA-I & Lysine (LYS) \\
\hline Homocystinuria & - & Methionine (MET) \\
\hline $\mathrm{N}$-acetyl-glutamate synthetase deficiency & & NAGS deficiency \\
\hline Carbamyl-phosphate synthetase I deficiency & & CPS deficiency \\
\hline Ornithine transcarbamoylase deficiency & & OTC deficiency \\
\hline
\end{tabular}


Argininosuccinate synthetase deficiency

Argininosuccinate lyase deficiency
AS deficiency

ASL deficiency

Arginase deficiency

IEAAM, inborn errors of amino acid metabolism; DSAA, disorder-specific amino acid(s). $\dagger$ Very rare IEAAM

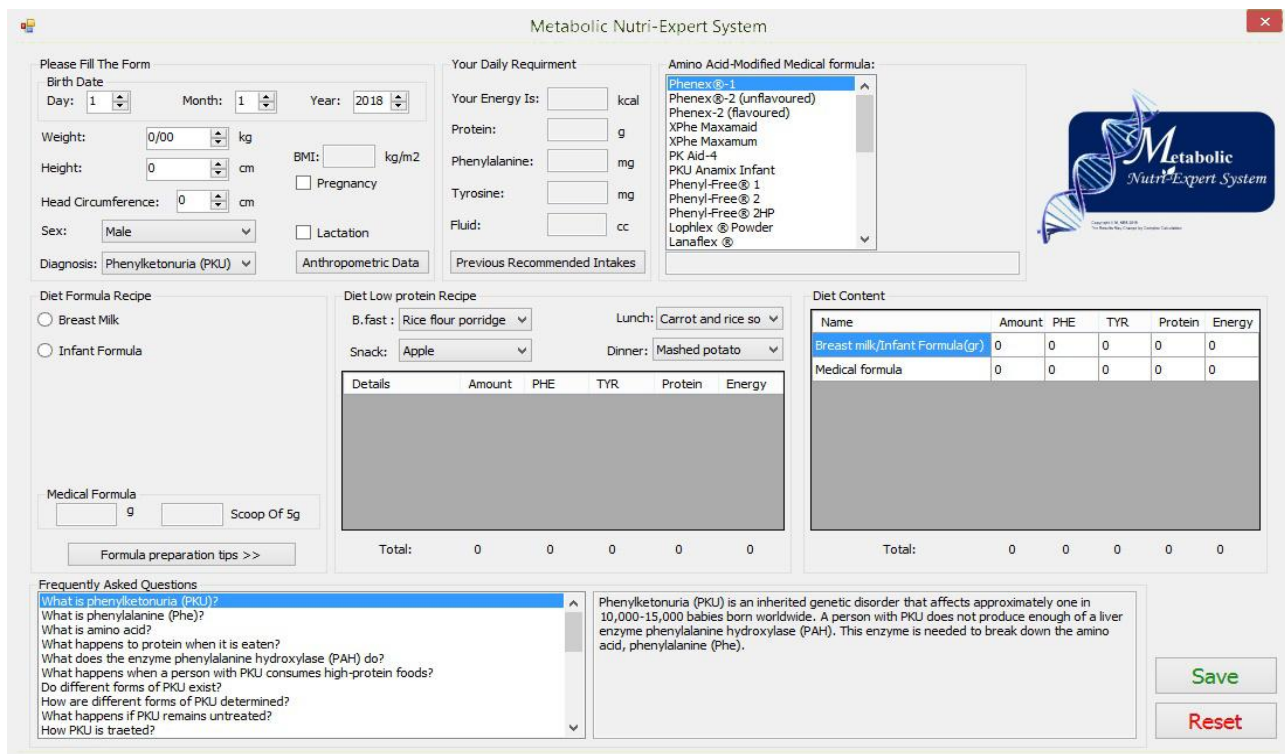

Fig 1. The whole schema of Metabolic Nutri-Expert System

\subsection{Anthropometric Assessment and Determination of Nutritional \\ Requirements}

To get recommended daily intakes (RDIs) of calorie and nutrients, the user has to import his/her anthropometric measurements (body weight, height and if indicated, i.e. for infants and toddlers, head circumference), diagnosis, and current blood concentration of DSAA. Daily requirements of energy, protein, DSAA, and fluids, recommended by the Genetic Metabolic Dietitians International (GMDI) nutrition guidelines, can be visualized to the user through user interface. In the case of lower or higher values of blood DSAA, the system alerts the user, represents the normal ranges of the parameter and addresses him/her to follow the preset protocol to overcome the acute phase of the disorder.

\subsection{Introduction of amino acid-modified medical formula}

Long-term administration of a low protein diet in nutrition management of IEAMM necessitates application of DSAAfree medical foods. Choosing any of the age-specific medical formula, with either breast milk or the selected infant formula (for those under 2 years of age), the 2 components of the proposed expert system (i.e. the knowledge base and interference engine) work together to recommend the required amounts of each (See Figure 2 and Figure 3). For 6-12 month old patients, recommendations for safe preparation of the formula and introduction of appropriate complementary nutrition are also given (See Figure 3)

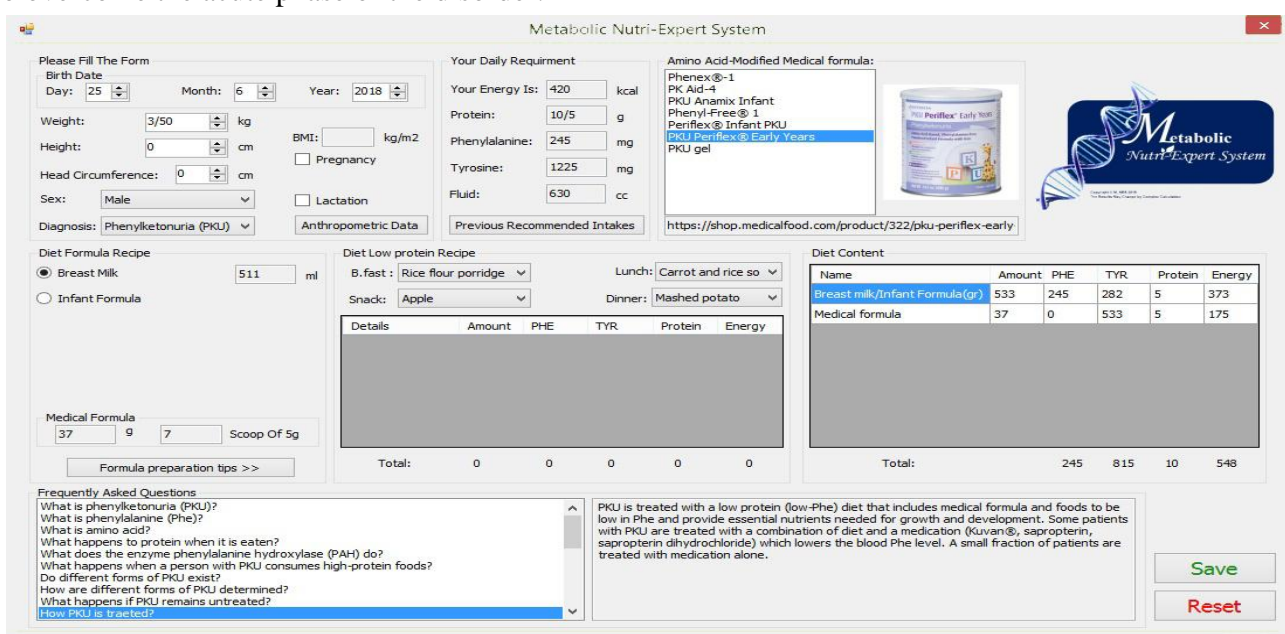

Fig 2. An example of diet calculation using Metabolic Nutri-Expert System for a 15 day-old male infant diagnosed with PKU. He weighs $3.5 \mathrm{~kg}$ and is decided to be placed on breast milk and PKU Periflex® Early Years medical formula 


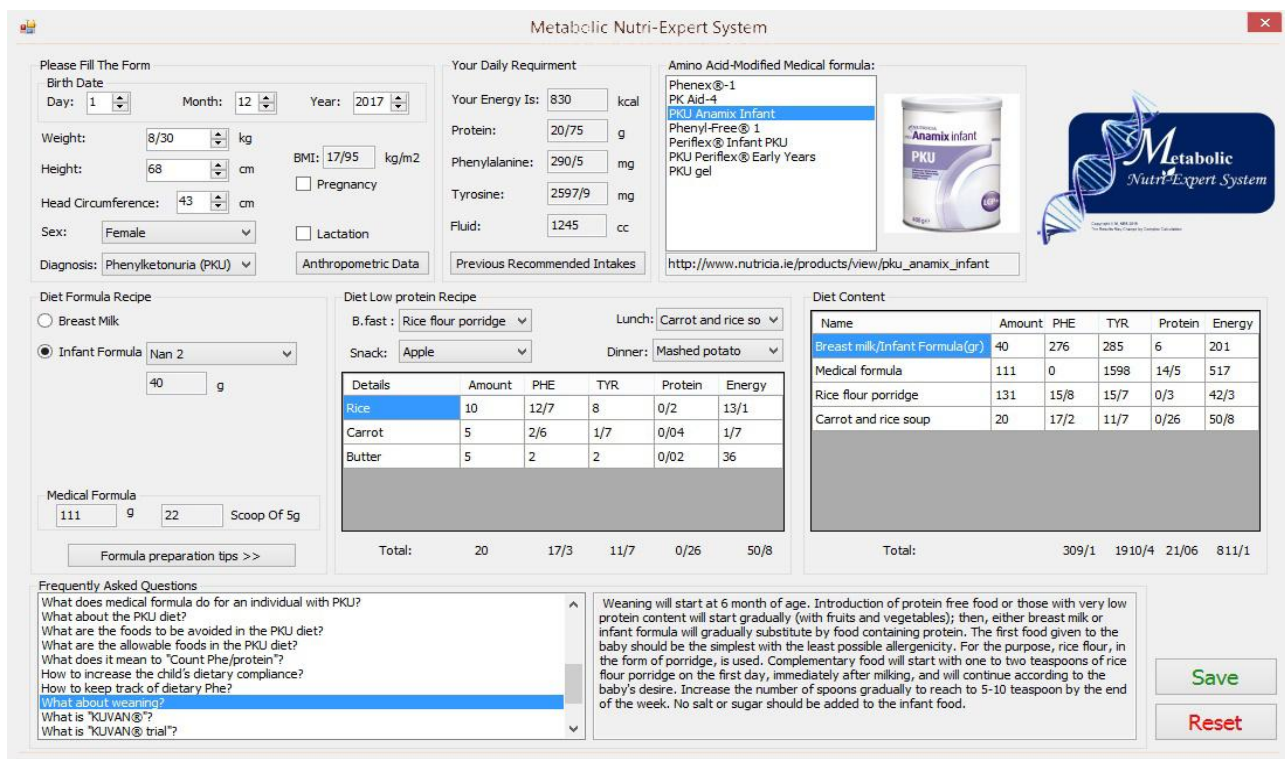

Fig 3. An example of diet prescription summery using Metabolic Nutri-Expert System for an older female infant (7 months old) weighing $8.3 \mathrm{~kg}$ who was diagnosed with PKU based on elevated blood PHE concentration at early infancy. She is decided to be placed on Nan-2 formula, PKU Anamix infant medical formula and complementary nutrition

For older patients, the Metabolic Nutri-Expert System calculates the amounts of dietary protein and DSAA, supplied by low protein recipes, as well as the recommended amount of age-specific medical food (See Figure 4).

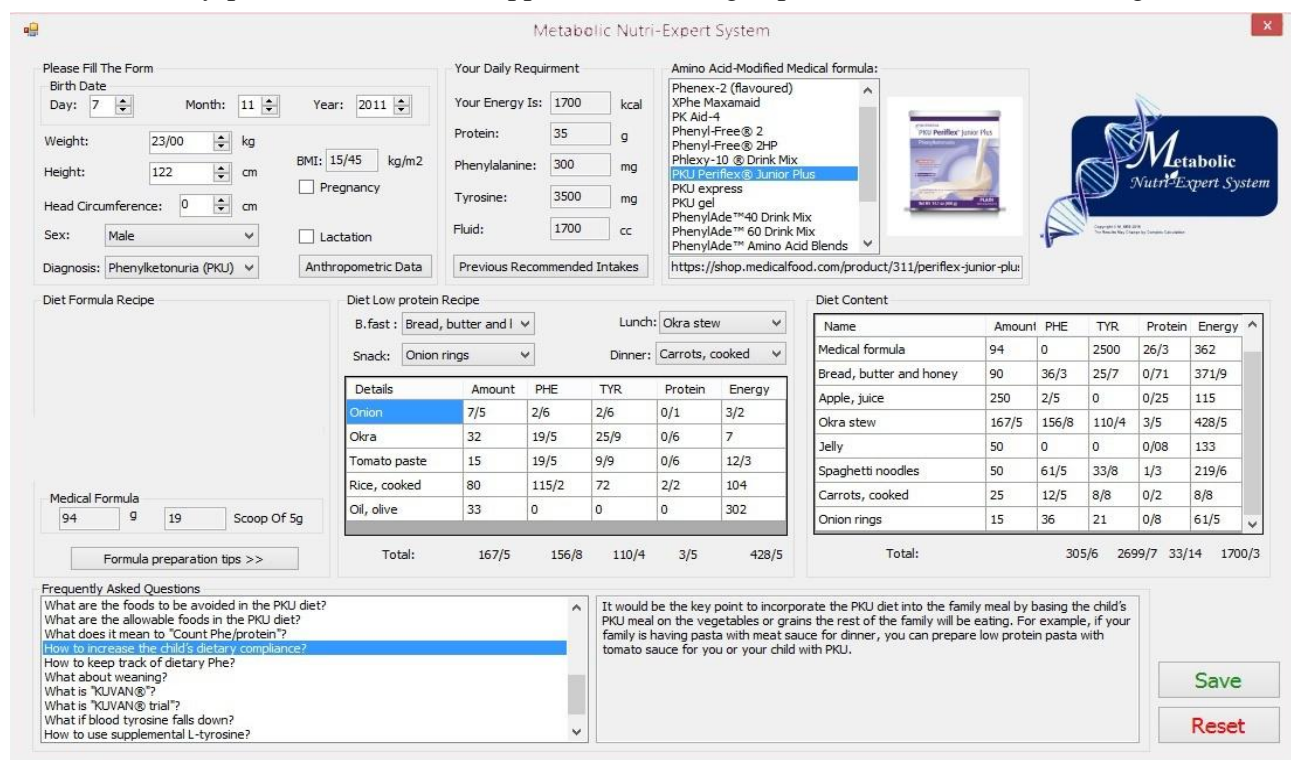

Fig 4. A diet calculation example using Metabolic Nutri-Expert System for a school-age child who was diagnosed with PKU. He weighs $23 \mathrm{~kg}$ is decided to be placed on PKU Periflex® Junior Plus PHE-free formula and a low protein (PHE-restricted) diet

\subsection{Low Protein Recipes based on Iranian and Modern Dietary Culture}

Since low protein diet reduces the production of toxic metabolites produced as a result of incomplete metabolism of amino acids, it is considered as the main component of IEAAM nutrition treatment [15].

In the next section of our proposed system, the patient can attain his/her dietary protein- and DSAA-requirements by various Iranian and modern low-protein recipes of cookery (breakfast, lunch, dinner, and snacks) offered by the expert system (Figures 3 and Figure 4). Metabolic Nutri-Expert System also allows the user to modify the diet as they like or make their own low protein recipes based upon the established standards.
Metabolic Nutri-Expert System calculates the protein-, DSAA-, and energy intakes of each meal and meals eaten throughout the day; moreover, it alerts when the protein-, DSAA-, and/or energy-content of the chosen meal is either lower or higher than the recommended values.

\subsection{Disease-related information}

The final section of Metabolic Nutri-Expert System is dedicated to increasing the knowledge and nutritional skills of the patient or his/her parent/caregiver about the disease and particular occasions. Instruction on what to do in special situations (ex: sick day management, acute illness, conditionally essential amino acid deficiency) can emerge in the form of dialog box by choosing the relevant question (Figure 5). 


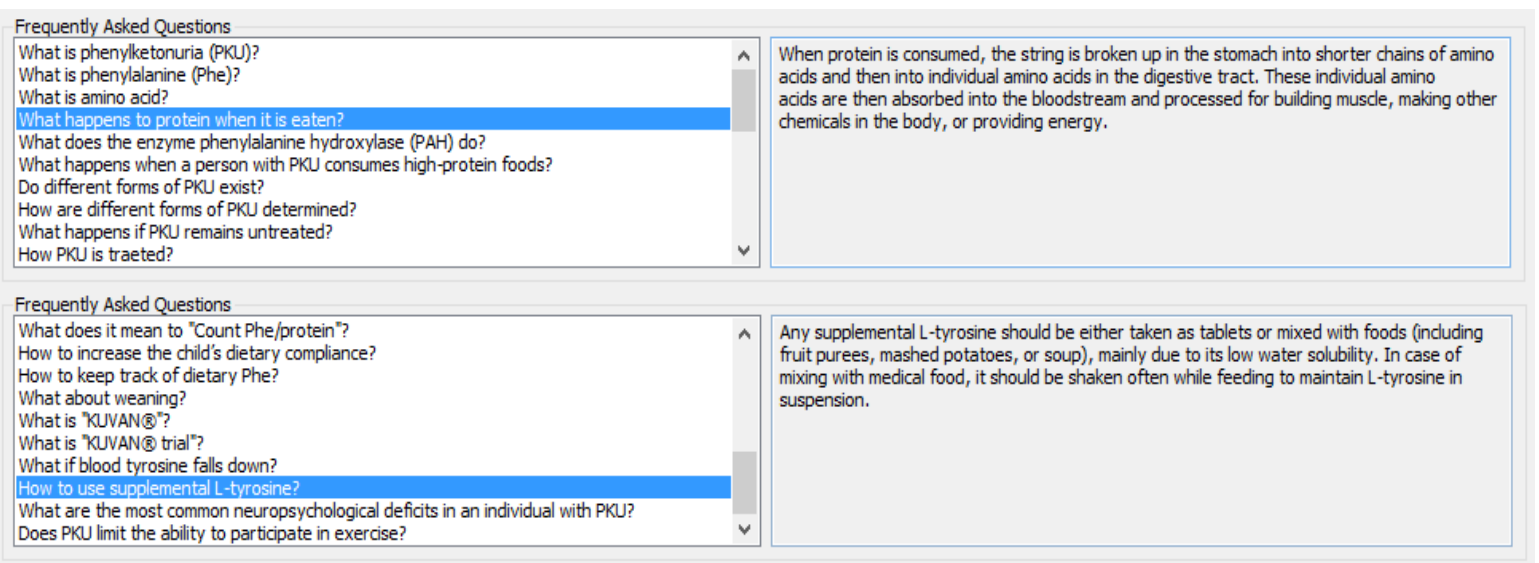

Figure 5: Disease-related information for increasing the knowledge and nutritional skills of the user a. An example of general information b. An example of disorder-specific nutritional information

\section{CONCLUSION}

The present expert system is proposed for either patient (his/her parent/caregiver) or metabolic dietitian/nutritionist to increase awareness on these rare disorders and to cope with the most important challenge of achieving proper metabolic control (i.e. prescription of a diet for various types of IEAAM) to lower the risk of acute metabolic decompensation and sudden death. The type of IEAAM, blood concentration of DSAA, and anthropometrical values are the inputs of the system to attain proper dietary recommendation.

The future scope of the idea of this paper is to improve selfmanagement of different IEAAM and facilitate achieving adequate metabolic control. Although there was an attempt to design a user-friendly expert system, limited computer-skill of the users may confine its use. However, encompassing both national and modern low protein recipes makes the proposed system more acceptable.

\section{ACKNOWLEDGMENTS}

This study was a complementary study of Ph.D. thesis in nutrition sciences (by Seyedeh Maryam Abdollahzadeh) and was entirely financed by Shiraz University of Medical Sciences (project no.95-01-87-13558). The authors would like to thank Center for Development of Clinical Research, Nemazee Hospital, Shiraz, Iran and Dr. Nasrin Shokrpour for editorial assistance.

\section{REFERENCES}

[1] El-Hattab, A.W., Inborn errors of metabolism. Clinics in perinatology, 2015. 42(2): p. 413-439.

[2] Hadj-Taieb, S., et al., Aminoacidopathies and organic acidurias in Tunisia: a retrospective survey over 23 years. La Tunisie Medicale, 2012. 90(3): p. 258-261.

[3] Ho, G., et al., Metabolic Diet App Suite for inborn errors of amino acid metabolism. Molecular genetics and metabolism, 2016. 117(3): p. 322-327.

[4] Raghuveer, T.S., U. Garg, and W.D. Graf, Inborn errors of metabolism in infancy and early childhood: an update. Am fam physician, 2006. 73(11): p. 1981-90.

[5] Ramaswamy, M., et al., A Rare Case of Malonic Aciduria Diagnosed by Newborn Screening in Qatar. International Journal of Neonatal Screening, 2017. 3(1): p. 5.
[6] Campa, K.M., M.A. Lloyd-Puryearb, and K.L. Huntingtonc, Nutritional Treatment for Inborn Errors of Metabolism: Indications, Regulations, and Availability of Medical Foods and Dietary Supplements Using Phenylketonuria as an Example. Mol Genet Metab, 2012. 107(1-2): p. 3-9.

[7] Bernstein, L.E., F. Rohr, and J.R. Helm, Nutrition Management of Inherited Metabolic Diseases. 2015: Springer.

[8] García, d.D.L., M. Cuervo, and J. Martinez, Software for performing a global phenotypic and genotypic nutritional assessment. Nutricion hospitalaria, 2013. 28(5): p. 1622 1632.

[9] Wysocki, T. and L. Gavin, Paternal involvement in the management of pediatric chronic diseases: Associations with adherence, quality of life, and health status. Journal of Pediatric Psychology, 2005. 31(5): p. 501-511.

[10] Abi-Wardé, M.-T., et al., Long-term metabolic follow-up and clinical outcome of 35 patients with maple syrup urine disease. Journal of inherited metabolic disease, 2017. 40(6): p. 783-792.

[11] Sweeney, A.L., R.M. Roberts, and J.M. Fletcher, Dietary protein counting as an alternative way of maintaining metabolic control in phenylketonuria, in JIMD ReportsCase and Research Reports, 2011/3. 2011, Springer. p. 131-139.

[12] ten Hoedt, A.E., et al., " MY PKU": increasing selfmanagement in patients with phenylketonuria. A randomized controlled trial. Orphanet journal of rare diseases, 2011. 6(1): p. 48.

[13] Wendel, U. and U. Langenbeck, Towards selfmonitoring and self-treatment in phenylketonuria-a way to better diet compliance. European journal of pediatrics, 1996. 155(1): p. S105-S107.

[14] Park, Y., H.S. Jeong, and N. Joo, A study on multicultural family wives adapting to Korean cuisine and dietary patterns. Nutrition research and practice, 2010. 4(5): p. 405-413.

[15] Dhananjayan, R., S. Rajeswari, and S. Swaminathan, Inborn metabolic disorders-An update. International Journal of Bioassays, 2015. 4(3): p. 3719-3728. 